\title{
Injury hospitalizations in Canada 2018/19
}

\author{
Xiaoquan Yao, MSc; Robin Skinner, MSP; Steven McFaull, MSc; Wendy Thompson, MSc
}

Tweet this article

\begin{abstract}
National injury hospitalization statistics are essential for understanding the burden and pattern of injuries. This paper used the Discharge Abstract Database to analyse injury hospitalizations in Canada (excluding Quebec) for fiscal year 2018/19. The results show that unintentional injuries were the eighth leading cause of hospitalization compared to all other diseases and conditions. For unintentional injury-related hospitalizations, in rank order, the leading causes were falls, suffocation, motor vehicle traffic crashes, poisonings, struck by/against, and fire/hot object/smoke. However, the rankings were different across age groups.
\end{abstract}

Keywords: injury hospitalizations, leading causes, unintentional injuries, self-inflicted injuries, assault, falls

\section{Introduction}

An injury is defined as the transfer of energy to human beings at rates and in amounts above or below the tolerance of human tissue. ${ }^{1,2}$ Injuries can be grouped according to external cause, which is a rough representation of the main energy types: falls (mechanical); motor vehicle traffic crashes (MVT) (mechanical); struck by/against (mechanical); poisonings (chemical); suffocation (asphyxiation, too little energy); fire/hot object/smoke (thermal/ chemical); and others (various energy types).

Injuries can also be classified as either unintentional or intentional. Unintentional injuries are those not caused on purpose or with intention to harm such as when someone trips and falls or is involved in a traffic accident. ${ }^{3,4}$ Intentional injuries result from a deliberate act of harm to oneself (self-inflicted) or another person (assault). ${ }^{3}$ When the intent is unclear, the injury is classified as undetermined intent. The intent and external cause provide insights into the mechanism of injuries that are fundamental for injury prevention.
Injuries are a public health concern, claiming 4.9 million lives worldwide in $2016^{5}$ and resulting in many more hospitalizations, emergency department visits and doctors' appointments. ${ }^{6}$ In Canada in 2018, 17843 people died from injuries, ${ }^{7}$ and in fiscal year $2017 / 18$, there were more than 269000 injury-related hospitalizations. ${ }^{8}$ The economic burden associated with injuries in 2010 was around CAD 27 billion. $^{9}$

Establishing a broad understanding of current injury burden and pattern in Canada is foundational for injury prevention efforts. Parachute, a national injury prevention organization, uses such information to set its strategic priority areas ${ }^{10}$ and to form the basis for reports on economic burden and cost of injury. ${ }^{9}$ Our 2019 paper showed that unintentional injuries were the first or second leading cause of death among 1-44 year olds and suicide was the second leading cause for those aged 15-34 years. ${ }^{11}$

Hospitalization statistics are essential to understanding injury burden including nonfatal events. Injury and trauma emergency

\section{Highlights}

- National injury hospitalization statistics are essential for understanding the burden and pattern of injuries, including non-fatal events, in Canada and informing prevention strategies.

- In fiscal year 2018/19, unintentional injuries were the eighth leading cause of hospitalizations overall compared to all other diseases and conditions. They were ranked ninth or higher among causes of hospitalization for every age group except children aged less than 1 year old.

- Falls were the leading cause of hospitalization in every age group for unintentional injury-related hospitalizations.

department and hospitalization statistics for fiscal year $2017 / 18$, published by the Canadian Institute for Health Information (CIHI), provide Canadian injury hospitalization rates by province/territory and hospitalization counts for specific injuries based on cause and intent. ${ }^{8}$ In a 2013 study, the Public Health Agency of Canada (PHAC) presented national injury hospitalization statistics from another perspective: PHAC ranked the hospitalizations for certain injury groupings (unintentional, self-inflicted and assault) compared to other diseases or conditions. ${ }^{12}$ That study also compared the hospitalizations associated with major external causes of injuries. ${ }^{13}$ Presenting the data in this way allows a clear understanding of the relative burden of the major injury groupings, which can complement the CIHI statistics. 
The objective of this paper is to update the previous tables using the most current data available for Canada (2018/19, Quebec excluded). The information presented in this paper is intended to illustrate the burden and pattern of injury-related hospitalizations to inform injury-prevention initiatives.

\section{Methods}

Our data source was the Discharge Abstract Database (DAD) 2018/19 from CIHI, which does not include Quebec hospitals. For this study, we selected only discharges from acute inpatient institutions, which we refer to as hospitalizations. A total of 2587663 acute inpatient records with a discharge date between 1 April 2018 and 31 March 2019 were kept after excluding stillbirths, cadavers and duplicates. The number of records represented the discharge count, not the number of individual patients.

The diagnoses in DAD 2018/19 were coded in ICD-10-CA (the International Statistical Classification of Diseases and Related Health Problems, 10th Revision, Canada). ${ }^{14}$ To compare the burden of unintentional injuries, self-inflicted injuries and assault to other diseases or conditions, we used the most responsible diagnosis (MRD) variable to determine the cause of a hospitalization. If a record's MRD starts with an S or $\mathrm{T}$, the record is defined as an injury record and further classified into unintentional injuries, selfinflicted injuries, assault, injuries with undetermined intent or others based on the external cause code in the diagnoses. If multiple external causes were found for one record, the classification was assigned following a priority order as assault, selfinflicted injuries, unintentional injuries, injuries with undetermined intent and others.

We conducted additional analyses for injury-related hospitalizations. To capture all hospitalization cases associated with injuries, we searched all external cause codes regardless of $\mathrm{S}$ or $\mathrm{T}$ code in MRD. We classified injuries based on intent and then external cause for unintentional injuries (falls, suffocation, MVT, poisonings, struck by/against, fire/hot object/smoke). We did not include complications of medical and surgical care; these were not the focus of this paper because their nature and prevention measures differ from that of most injuries. ${ }^{3}$ If a record was associated with multiple injury groups, it was counted multiple times.

We used SAS Enterprise Guide version $7.1^{15}$ to compile the pooled and stratified (by sex and age) counts. The population estimates (Quebec excluded) on 1 October 2018 from Statistics Canada ${ }^{16}$ were used for crude rate calculation.

\section{Results}

\section{Leading causes of all hospitalizations}

The leading causes of all hospitalizations are presented in Table 1. Compared to all other diseases and conditions (including hospitalizations due to examinations, specific care, potential hazards and reproduction-related circumstances and not classifiable symptoms, signs and findings), unintentional injuries ranked eighth overall, seventh among males and ninth among females. For every age group except less than 1 year olds, unintentional injuries were ranked ninth or higher. They were among the top five for 1-34 year olds and those aged 80 years and over. Self-inflicted injuries were the ninth leading cause of hospitalizations for 15-19 year olds and tenth for 20-24 year olds.

\section{Leading causes of injury-related hospitalizations}

Table 2 shows that overall, males and females had similar rank order in hospitalization rates for unintentional injuries, but diverged for intentional injuries. Females displayed higher rates of hospitalizations associated with unintentional falls and self-inflicted injuries than males. In contrast, males showed higher rates of hospitalizations associated with unintentional suffocation, MVT, poisonings, struck by/ against, fire/hot object/smoke and assault.

For all ages combined, the rankings of unintentional injuries by external causes (from highest to lowest) were falls, suffocation, MVT, poisonings, struck by/ against and fire/hot object/smoke. Across the lifespan, the rate of hospitalization associated with falls increased sharply among those aged 65 years and over, jumping to 4 times and then 16 times as much as 45-64 year olds.

Aside from falls as the leading cause of hospitalizations associated with unintentional injuries in every age group, we can see variations in the ranking of other external causes across age groups.
Suffocation was the second leading cause for less than 10 year olds and those aged 45 years and over. MVT was the second leading cause for 15-44 year olds. Struck by/against was second for those aged 10-14 years.

Of note is that 15-24 year olds displayed a higher rate of hospitalization associated with self-inflicted injuries than unintentional falls. Those aged 20-24 years showed the highest rate of hospitalizations associated with assault.

\section{Discussion}

This paper presents the most up-to-date national injury hospitalization statistics to help understand the burden and pattern of injuries in Canada.

The results reveal that, overall, unintentional injuries were the eighth leading cause of hospitalizations in fiscal year $2018 / 19$. Falls were the leading cause of hospitalizations related to unintentional injury across all age groups, particularly among seniors (65 years and over), with the rate jumping to 4 times and then 16 times as much as in middle age. The burden of seniors' falls on the Canadian health care system is substantial. ${ }^{9}$ Moreover, the aging of the baby-boom cohort increases the proportion of the population aged 65 and over. Ongoing surveillance of falls in this population is very important to understand the trend and develop effective prevention programs. ${ }^{17}$ The variations in the ranking of other unintentional injury groups across age groups highlight the significance of tailoring prevention efforts to specific age groups. They are the base for strategic planning and public messaging in injury prevention. ${ }^{10,18}$

Self-inflicted injuries and assaults among young people are also an important public health concern. Self-inflicted injuries are used as a proxy for suicide attempts, and the high rate of hospitalizations associated with self-inflicted injuries among 15-24 year olds warrants further research. The group showing the highest rate of hospitalizations associated with assault were aged 20-24 years. These facts highlight the opportunity for prevention efforts among youth and young adults. ${ }^{19,20}$

\section{Limitations}

The population of Quebec constitutes $22.6 \%$ of the Canadian population. ${ }^{16}$ Our 


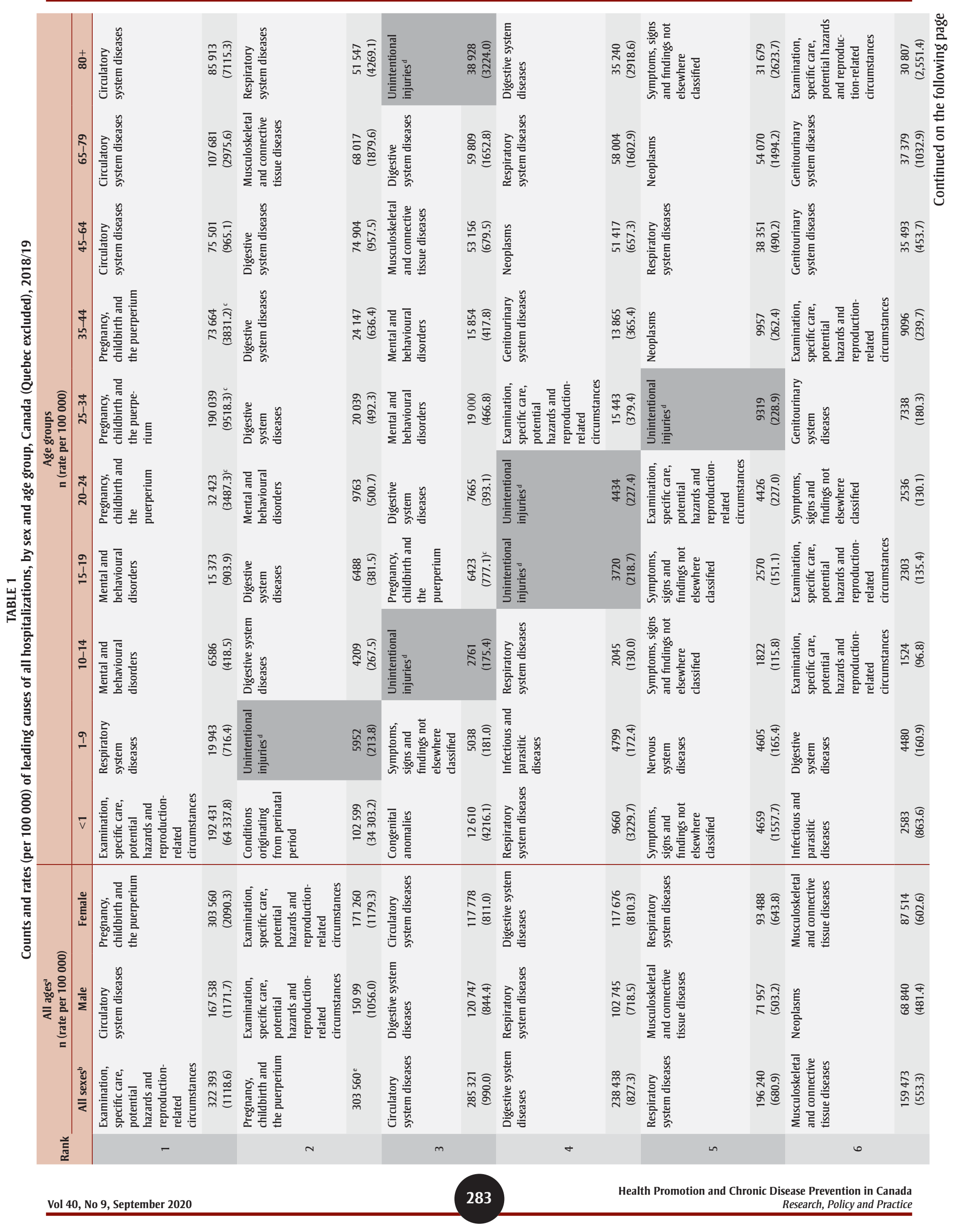




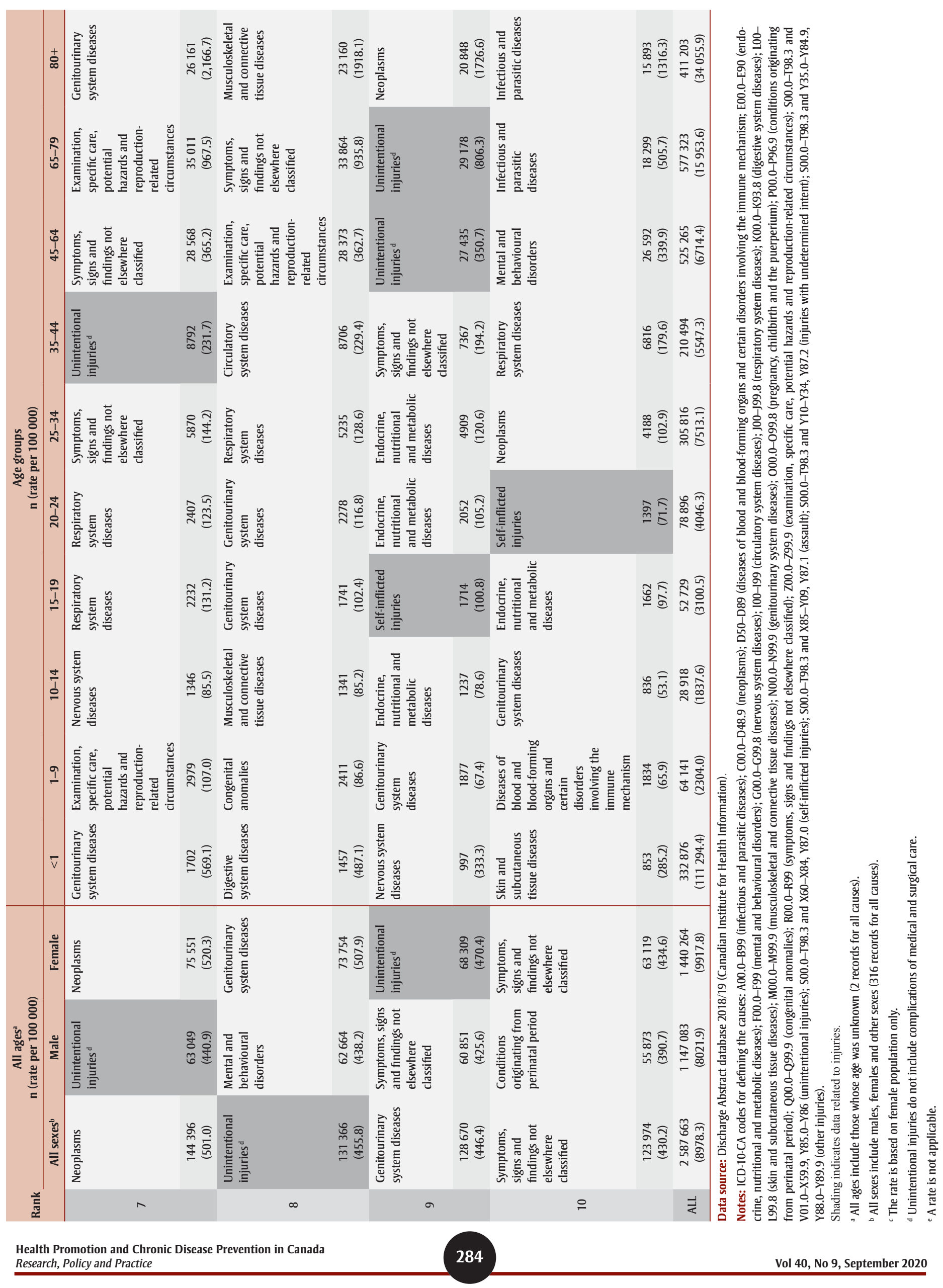




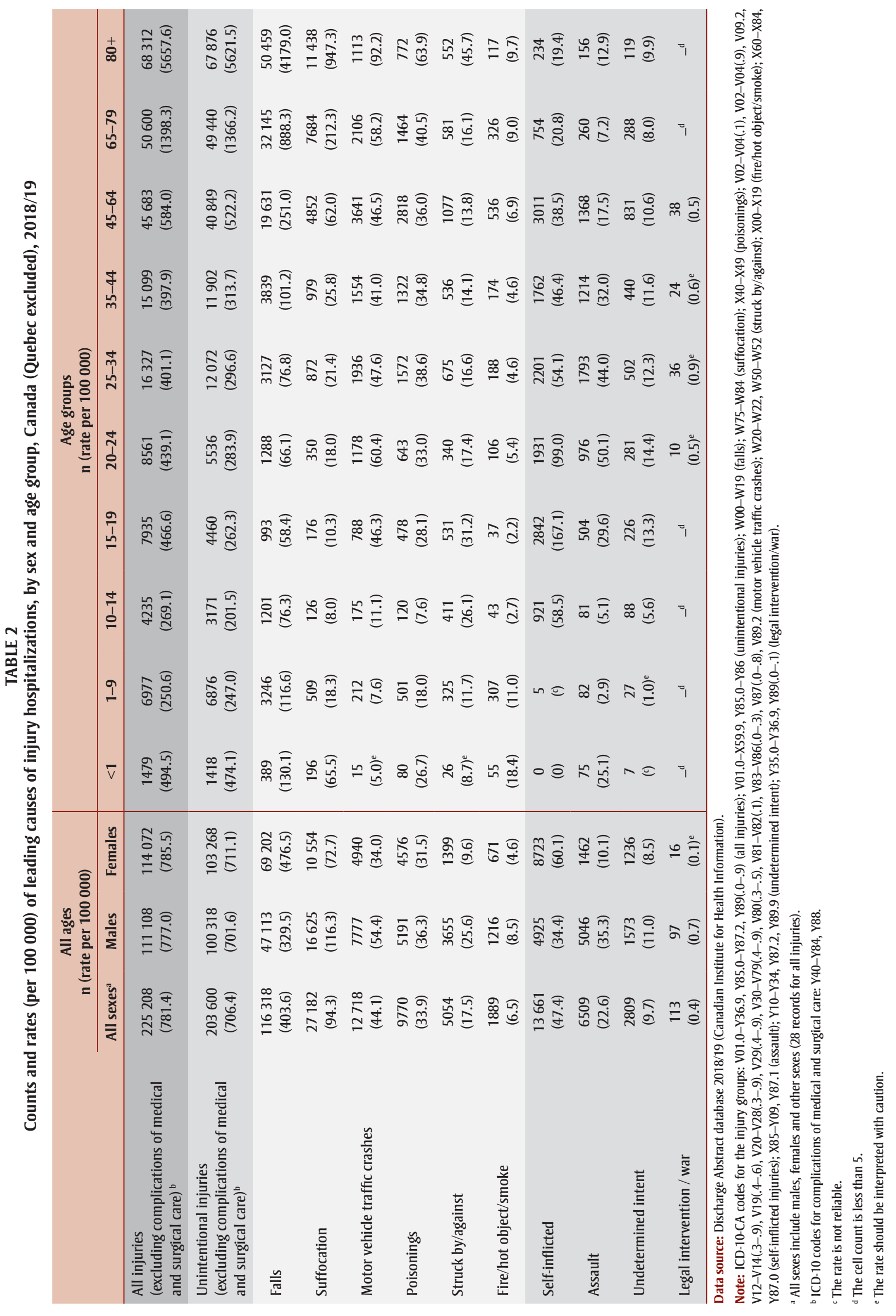


data source was the DAD from CIHI, which does not include Quebec hospitalization data. The Quebec Ministry of Health and Social Services provided hospitalization statistics by sex (no age group breakdown) through MED-ÉCHO, ${ }^{21}$ while our analyses require micro-level data.

Second, to compare the burden of injuries with other diseases and conditions, we used both MRD ( $\mathrm{S}$, T codes) and external cause codes to identify injury cases. This method classifies records whose external cause was the underlying cause for their non-injury MRD into non-injury cases. Therefore, it undercounts the injury cases. It also indicates that we should not expect the equal numbers of unintentional, selfinflicted and other injuries between Table 1 and 2 .

In addition, the method of data analysis can affect ranking. To accurately monitor the trend in injury hospitalizations, consistent case definitions and procedures to compile and report data are necessary. We aim to do more work in this area.

\section{Conclusion}

Overall, unintentional injuries were the eighth leading cause of hospitalization among all causes. For unintentional injury-related hospitalizations, in rank order, the leading causes were falls, suffocation, MVT, poisonings, struck by/against, and fire/hot object/smoke. However, the rankings were different across age groups. The updated injury hospitalization information is critical for understanding the burden and pattern of injuries in Canada.

\section{Acknowledgements}

The authors thank Stephanie Cowle and Pamela Fuselli at Parachute for their valuable comments.

\section{Conflicts of interest}

All authors declare no conflicts of interest.

\section{Authors' contributions and statement}

All authors have read and approved of the content of this article. XY was involved in conceptualization, data analysis, interpretation and manuscript preparation. RS, SM and WT were involved in conceptualization, data interpretation and manuscript preparation.
The content and views expressed in this article are those of the authors and do not necessarily reflect those of the Government of Canada.

\section{References}

1. Haddon W Jr, Suchman EA, Klein D. Accident research: methods and approaches. New York (NY): Harper and Row; 1964.

2. Robertson LS. Injury epidemiology: research and control strategies. Third edition. New York (NY): Oxford University Press; 2007.

3. Public Health Agency of Canada. Injury in review, 2012 edition: spotlight on road and transport safety. Ottawa (ON): Public Health Agency of Canada; 2012.

4. Chen Y, Mo F, Yi QL, Jiang Y, Mao Y. Unintentional injury mortality and external causes in Canada from 2001 to 2007. Chronic Dis Inj Can. 2013; 33(2):95-102.

5. World Health Organization (WHO). The top 10 causes of death [Internet]. Geneva (CH): WHO; 2018 [cited 2019 Nov 21]. Available from: https:// www.who.int/news-room/fact-sheets /detail/the-top-10-causes-of-death

6. World Health Organization (WHO) Health topics: injuries [Internet] Geneva (CH): WHO; 2018 [cited 2019 Nov 21]. Available from: http://www .who.int/topics/injuries/en/

7. Statistics Canada. Table 13-10-0156-01 Deaths, by cause, Chapter XX: External causes of morbidity and mortality (V01 to Y89). Ottawa (ON): Statistics Canada; 2019 [cited 2019 Nov 21]. Available from: https:// www150.statcan.gc.ca/t1/tbl1/en/tv .action?pid $=1310015601$

8. Canadian Institute for Health Information. Injury and trauma emergency department and hospitalization statistics, 2017-2018 [Internet]. Ottawa (ON): Canadian Institute for Health Information; 2019 [cited 2019 Nov 21]. Available from: https://www.cihi.ca /en/access-data-reports/results?f\% 5B0 $\% 5 \mathrm{D}=$ field_primary_theme $\% 3 \mathrm{~A} 2048$
9. Parachute. Cost of injury in Canada [Internet]. Toronto (ON): Parachute; 2015 [updated 2019 May 31; cited 2019 Nov 21]. Available from: https:// parachute.ca/en/professional-resource /cost-of-injury-report/

10. Parachute. Our leap forward: Parachute's strategic plan, 2018-2020. Toronto (ON): Parachute [cited 2019 Nov 21]. Available from: https://parachute.ca /en/about-us/our-leap-forward -parachutes-strategic-plan-2018-2020/

11. Yao X, Skinner R, McFaull S, Thompson W. At-a-glance: 2015 injury deaths in Canada. Health Promot Chronic Dis Prev Can. 2019;39(6-7):225-31. doi: 10.24095/hpcdp.39.6/7.03.

12. Public Health Agency of Canada. Leading causes of hospitalizations, Canada, 2009/10, males and females combined, counts (age-specific hospitalization rate per 100,000$)$. Ottawa (ON): Public Health Agency of Canada. Available from: https://www.canada .ca/en/public-health/services/reports -publications/leading-causes-death -hospitalization-canada/2009-10 -males-females-combined-counts -specific-hospitalization-rate.html

13. Public Health Agency of Canada. Leading causes of injury hospitalizations, Canada, 2009/10, counts (agespecific hospitalization rate per 100,000). Ottawa (ON): Public Health Agency of Canada; 2013. [Cat No.: HP32-6/2010E-PDF.]

14. Canadian Institute for Health Information. Codes and classifications [Internet]. Ottawa (ON): Canadian Institute for Health Information [cited 21 Nov 2019]. Available from: https:// www.cihi.ca/en/submit-data-and - vi e w - s t a nd a rd s / c o d e s - a nd -classifications

15. SAS Institute Inc. SAS Enterprise Guide 7.1. Cary (NC): SAS Institute Inc.

16. Statistics Canada. Canadian population estimates by age, sex, and provinces and territories on Oct 1, 2018. Ottawa (ON): Statistics Canada; 2019. 
17. Public Health Agency of Canada. Seniors' falls in Canada: second report. Ottawa (ON): Public Health Agency of Canada; 2014. Available from: https://www.canada.ca/content/dam /phac-aspc/migration/phac-aspc /seniors-aines/publications/public /injury-blessure/seniors_falls-chutes_ aines/assets/pdf/seniors_falls-chutes _aines-eng.pdf

18. Parachute. Injury topics; Discover how to prevent injuries before they happen [Internet]. Toronto (ON): Parachute; 2019 [cited 2019 Nov 21]. Available from: https://parachute.ca /en/injury-topics/

19. Canada Suicide Prevention Service. CSC/CSPS public fact sheet [Internet]. Crisis Services Canada; 2017 [cited 2019 Nov 21]. Available from: https:// www.crisisservicescanada.ca/en/fast -facts/

20. Public Safety Canada. Crime prevention inventory. Ottawa (ON): Public Safety Canada; 2018 [modified 2018 Aug 21; cited 2019 Nov 21]. Available from: https://www.publicsafety.gc.ca /cnt/cntrng-crm/crm-prvntn/nvntr /index-en.aspx?t $=2 \&$ SORT $=$ Title $\& B Y=$ ASC

21. Quebec Ministry of Health and Social Services. MED-ÉCHO - Hospitalisations et chirurgies d'un jour dans les centres hospitaliers du Québec. Québec (QC): Santé et services sociaux Québec; 2019 [cited 2019 Nov 21]. Available from: https://www.msss.gouv.qc.ca /professionnels/statistiques-donnees -services-sante-services-sociaux/med -echo-hospitalisations-et-chirurgies-d -un-jour-dans-les-centres-hospitaliers -du-quebec/ 